\title{
Genotype distribution and the relative risk factors for human papillomavirus in Urumqi, China
}

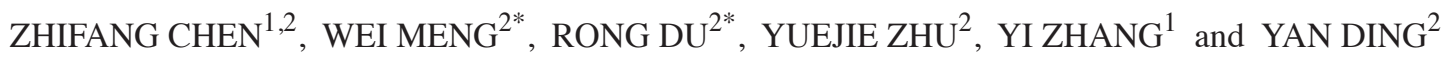 \\ ${ }^{1}$ Xiangya Hospital, Central South University, Changsha, Hunan 410000; ${ }^{2}$ Gynecology Department, \\ First Affiliated Hospital of Xinjiang Medical University, Urumqi, Xinjiang 830054, P.R. China
}

Received January 21, 2013; Accepted April 11, 2013

DOI: $10.3892 /$ etm.2013.1073

\begin{abstract}
The aim of this study was to investigate human papillomavirus (HPV) infection and HPV genotype distributions in Urumqi, Xinjiang, China. The related risk factors for high-risk HPV infection was also analyzed. A stratified cluster sampling method was used for the population-based cervical cancer screening of women aged 18-69 years in the Urumqi Saybagh district. Exfoliated cervical cell samples were collected for liquid-based cytology detection and HPV genotyping DNA microarrays. Education level, number of sexual partners, condom use and occupation were used in the multivariate analysis model. The HPV infection rate of women working in service industries was significantly higher compared with those of white-collar workers, community residents and migrant workers. The 35-44-year-old migrant worker group had the highest HPV infection rates among all of the groups in the three different age ranges. The number of marriages, education level, smoking history, number of abortions, use of condoms, number of sexual partners, number of sexual partners in the past five years and occupation were all associated with female HPV infection rate $(\mathrm{P}<0.05)$. The 35-44-year-old women were the age group with the highest HPV infection rate. The HPV infection rate of females in service industries was the highest. Education level and condom use were protective factors of HPV infection, while the number of sexual partners and occupation were risk factors for HPV infection.
\end{abstract}

\section{Introduction}

Cervical cancer is one of the most common female malignancies. In certain developing countries, cervical cancer has the highest incidence; however, in North America and Europe,

Correspondence to: Dr Yan Ding, Gynecology Department, First Affiliated Hospital of Xinjiang Medical University, 393 Xinyi Road, Urumqi, Xinjiang 830054, P.R. China

E-mail: 2332917439@qq.com

*Contributed equally

Key words: human papillomavirus, genotype distribution, cervical cancer the incidence is far lower than the incidences of breast cancer, endometrial cancer and ovarian cancer. According to the estimates of the International Cancer Research Center, there are $\sim 371,200$ new cases of cervical cancer, accounting for $\sim 9.8 \%$ of all tumors and $15 \%$ of female cancers annually (1). Of them, $78 \%$ of new cases appear in developing countries, while only $\sim 4.4 \%$ occur in developed countries. Annually, there are $~ 100,000$ new cases of cervical cancer in China, accounting for $1 / 4$ of the global incidence of cervical carcinoma and ranking first in the prevalence of gynecological malignancies (2). In certain developing countries, cervical cancer is the leading cause of mortality for females. In developed countries, the incidence is significantly reduced. In certain countries, the mortality rate of cervical cancer has been reduced by $>50 \%$ as a result of the early diagnosis and treatment of cervical precancerous lesions $(3,4)$. A number of studies have demonstrated that cervical cancer is associated with human papilloma virus (HPV) infection, particularly the high-risk HPV genotypes (5-7).

At present, a HPV genotyping assay has been adopted in developed regions as a main method for screening cervical lesions. A HPV vaccine is currently the first choice for the prevention of HPV infection and consequent reduction of the incidence of cervical cancers. Research on HPV vaccines is mainly targeted at high-risk HPV genotypes. It is reported that persistent infection with HPV is the crucial factor for cervical cancer (8). To investigate the results of the HPV vaccine and HPV examination-based screening for cervical cancer prevention, HPV infection in large population requires examination.

A preliminary epidemiological study has investigated different genotypes of HPV infection in a number of regions in China (9). However, in Xinjiang, where the incidence of cervical cancer is highest, there have been no region-based population surveys of HPV infection. In the current study, the analysis of female HPV infection in the Urumqi district of Xinjiang was examined, including assessment of age and genotype distribution. This study provides scientific evidence for the HPV vaccine prevention plan and support for an effective cervical cancer prevention strategy.

\section{Subjects and methods}

Subjects. Females aged 18-69 years in the Urumqi Saybagh district were recruited. All patients provided informed consent. The subjects were divided into four groups, including 
543 migrant workers, 525 white-collar workers (government employees, employees in institutionalized organizations and managers in companies), 487 workers in service industries and 714 community residents with a total of 2,269 . By the stratified cluster random sampling method, age-stratified sampling was also applied in selected populations, with every 5 years as the age group. Prior written and informed consent were obtained from every patient and the study was approved by the ethics review board of First Affiliated Hospital of Xinjiang Medical University (Urumqi, China).

Instruments and reagents. A HPV nucleic acid amplification typing detection reagent kit was purchased from Cape Biochemistry Ltd., Co. (Guangdong, China). A HybriMax medical nucleic acid molecule rapid hybridization instrument, an Autocyte Prep system for liquid-based cytology, CytoRich Preservative fluid and an electronic colposcope were purchased from Shenzhen Goldway Industrial Inc. (Shenzhen, China).

$H P V$ detection. All subjects underwent gynecological examination and cervical secretion was collected. A genome extraction kit from Cape Biochemistry Ltd., Co. was used for DNA extraction. Polymerase chain reaction (PCR) amplification using the ABI7300 PCR amplification instrument (Cape Biochemistry Ltd. Co.) and hybridization were performed. Results were obtained by visual observation. A clear blue purple dot was considered positive. According to the film HPV genotype distribution map, the HPV subtype was determined. If the two control points were positive and other points were negative, DNA test results of HPV subtypes in the reagent kit were considered negative. If at least one HPV genotype point was positive, it demonstrated single or mixed HPV infection.

Liquid-based cytology test (LCT). An AutoCyte liquid-based thin-film machine (TriPath Imaging Inc., BD Diagnostics, Burlington, NC, USA) was used for automatic production and dyeing. Results were examined by optical microscopy and cytological diagnosis was in accordance with The Bethesda System (TBS, 2001) (10).

Colposcopy and pathological diagnosis. For all the subjects with abnormal LCT results, colposcopy and biopsy were performed. For positive high-risk HPV infection in cervical secretions with atypical squamous cells of undetermined sign and/or low-grade squamous intraepithelial lesions in the LCT or worse, colposcopy was performed again. Biopsy was performed by electronic colposcopy in suspicious lesions for pathological diagnosis.

Statistical analysis. VFP software was used for database building (Microsoft Corp, Redmond, WA, USA). SPSS 16.0 software (SPSS, Inc., Chicago, IL, USA) was used for statistical analysis. Data are presented as the mean \pm standard error of the mean (SEM). The HPV infection rates in different age groups with different occupations, cervical intraepithelial neoplasia (CIN; a premalignant cervical disease) rate comparison of different levels and cervical lesion HPV detection rate comparison of different degrees from pathological results were examined by $\chi^{2}$ test. Single factor analysis was performed for each factor and analyzed by the logistic regres- sion model. $\mathrm{P}<0.05$ was considered to indicate a statistically significant result.

\section{Results}

Detected rates of different HPV genotypes in cervical lesions of females of different ages. In this study, 2,269 females were enrolled with an average age of $39.65 \pm 0.203$ years, ranging from 18 to 69 years. The subjects included 543 migrant workers (individuals going to Urumqi region to work from the other areas of China) with an average age of $35.60 \pm 0.612$ years, 525 white-collar workers (government employees, employees in institutionalized organizations and managers in companies) with an average age of $34.90 \pm 0.319$ years, 487 workers in service industries with an average age of $32.78 \pm 0.364$ years and 714 community residents with an average age of $43.60 \pm 0.225$ years.

As shown in Table I, there were 460 positive cases of HPV, with a detection rate of $20.27 \%$ in the whole investigated group in this study. A total of 21 HPV genotypes were detected, including 13 high-risk genotypes: HPV-16, 18, 31, $33,35,39,45,51,52,56,58,59$ and 68 ; five low-risk genotypes: HPV-6, 11, 42, 43, 44; and three common genotypes of Chinese individuals: HPV-53, 56 and CP8304. For the females with infections of high-risk HPV genotypes, the high-risk HPV genotypes were mainly HPV-16, 58, 52 and 18, which accounted for $43.35,18.35,8.86$ and $7.59 \%$, respectively, of the total infection. The four genotypes accounted for $78.16 \%$ of the high-risk HPV genotypes.

As shown in Fig. 1A, the high-risk HPV-positive rates in the three age groups (18-34, 35-44 and 45-69 years) were $18.07,29.64$ and $7.64 \%\left(\chi^{2}=105.02, P<0.05\right)$, respectively. The differences in the high-risk HPV-positive rates in the three age groups were significant $\left(\chi^{2}=19.43, \mathrm{P}<0.05 ; \chi^{2}=22.62, \mathrm{P}<0.01\right.$; and $\chi^{2}=66.17, \mathrm{P}<0.01$, respectively).

$H P V$-positive rates are higher among the groups with deteriorating cervical lesions. To determine whether HPV infection is related to different degrees of cervical lesions, HPV infection was determined in the patients with different degrees of cervical lesions. As shown in Fig. 1B, the HPV-positive rate in normal groups without symptoms of cervical lesions was $\sim 9 \%$. The HPV-positive rates in chronic cervicitis and CIN I patients were $\sim 49$ and 70\%, respectively. The HPV-positive rates in CIN II and CIN III patients were $\sim 79$ and 95\%, respectively. However, the HPV-positive rate in cervical cancer patients was $\sim 100 \%$. These results suggest that HPV infection rates are increased in groups with deteriorating cervical lesions $\left(\chi^{2}=697.72, \mathrm{P}<0.05\right)$.

Comparison of the HPV infection rates among different occupation groups. The HPV infection rates in patients with different occupations were also investigated. As shown in Fig. 2, the 35-44-year-old migrant worker group had the highest HPV infection rates among all the groups in the three different age ranges. The HPV infection rate of females in service industries was significantly higher compared with those of the whitecollar workers, community residents and migrant worker groups $\left(\chi^{2}=74.46, \mathrm{P}<0.05 ; \chi^{2}=84.93, \mathrm{P}<0.05 ;\right.$ and $\chi^{2}=13.41, \mathrm{P}<0.05$, respectively) at all three age ranges. These results suggest that the 35-44-year-old migrant worker group and the service industry workers may have a higher risk of HPV infection. 
Table I. Distribution of HPV genotypes infecting females in Urumqi.

\begin{tabular}{|c|c|c|c|}
\hline HPV genotype & Total positive cases & Positive ratio of population $^{a}$ & Positive ratio of group ${ }^{a}$ \\
\hline \multicolumn{4}{|l|}{ High-risk } \\
\hline HPV-16 & 137 & $137 / 2269(6.03)$ & $137 / 316(43.35)$ \\
\hline HPV-18 & 24 & 24/2269 (1.05) & 24/316 (7.59) \\
\hline HPV-31 & 21 & $21 / 2269(0.93)$ & $21 / 316(6.65)$ \\
\hline HPV-33 & 13 & $13 / 2269(0.57)$ & $13 / 316(4.11)$ \\
\hline HPV-35 & 0 & 0 & 0 \\
\hline HPV-39 & 10 & $10 / 2269(0.44)$ & $10 / 316(3.16)$ \\
\hline HPV-45 & 5 & $5 / 2269(0.22)$ & $5 / 316(1.58)$ \\
\hline HPV-51 & 5 & $5 / 2269(0.22)$ & $5 / 316(1.58)$ \\
\hline HPV-52 & 28 & $28 / 2269(1.23)$ & 28/316 (8.86) \\
\hline HPV-56 & 5 & $5 / 2269(0.22)$ & $5 / 316(1.58)$ \\
\hline HPV-58 & 58 & $58 / 2269(2.56)$ & $58 / 316(18.35)$ \\
\hline HPV-59 & 0 & 0 & 0 \\
\hline HPV-68 & 10 & $10 / 2269(0.44)$ & $10 / 316(3.16)$ \\
\hline \multicolumn{4}{|l|}{ Low-risk } \\
\hline HPV-6 & 5 & $5 / 2269(0.22)$ & $5 / 14(35.71)$ \\
\hline HPV-11 & 3 & $3 / 2269(0.13)$ & $3 / 14(21.43)$ \\
\hline HPV-42 & 3 & $3 / 2269(0.13)$ & $3 / 14(21.43)$ \\
\hline HPV-43 & 3 & $3 / 2269(0.13)$ & $3 / 14(21.43)$ \\
\hline HPV-44 & 0 & 0 & 0 \\
\hline \multicolumn{4}{|c|}{ Common type for Chinese } \\
\hline HPV-53 & 10 & $10 / 2269(0.44)$ & $10 / 30(33.33)$ \\
\hline HPV-66 & 10 & $10 / 2269(0.44)$ & $10 / 30(33.33)$ \\
\hline HPV-CP8304 & 10 & $10 / 2269(0.44)$ & $10 / 30(33.33)$ \\
\hline Mixed infection & 100 & $100 / 2269(4.41)$ & $100 / 100(100.0)$ \\
\hline Total & 460 & & \\
\hline
\end{tabular}

${ }^{a}$ Values in brackets are percentages. HPV, human papillomavirus.
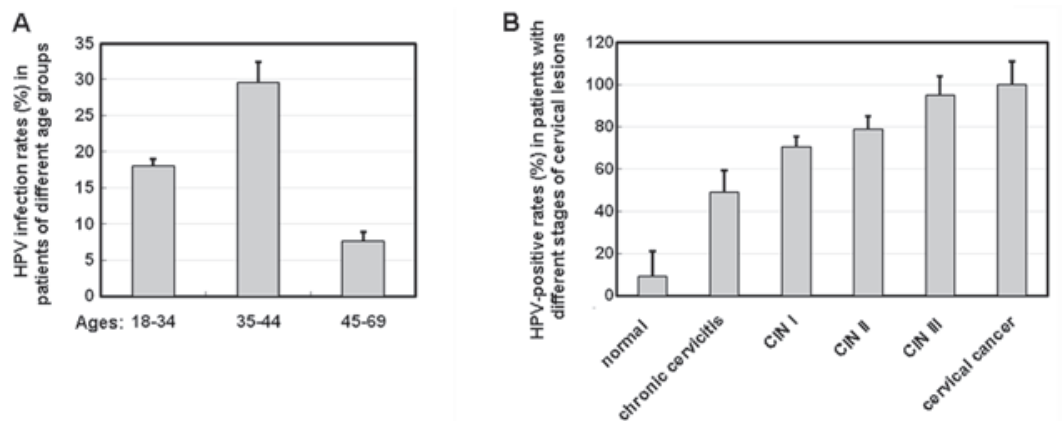

Figure 1. Human papillomavirus (HPV) genotype detection in females aged 18-69 years in Urumqi Saybagh district, China. All subjects underwent gynecological examination and cervical secretion was collected. A genome extraction kit was used for DNA extraction. Polymerase chain reaction (PCR) amplification using the ABI7300 PCR amplification instrument and hybridization were performed. For all the subjects with abnormal liquid-based cytology results, colposcopy and biopsy were performed. The data shown are mean \pm standard error of the mean (SEM). (A) HPV infection rates in patients in different age groups. (B) HPV-positive rates in patients with different stages of cervical lesions. Cervical intraepithelial neoplasia (CIN) is a premalignant cervical disease, which includes 3 stages: CIN I, CIN II and CIN III, with increasing disease severity.

Analyses of the high-risk HPV infections. To investigate the factors related to the HPV infections, single analysis was performed to study the related factors by comparing data between the HPV-positive and HPV-negative groups. As shown in Table II, several factors, including the number of marriages, education level, smoking history, number of abortions, condom use, number of sexual partners and number of sexual partners within the last 5 years, as well as occupation were significantly different between the two groups $(\mathrm{P}<0.05$; Table II). This analysis indicates that the number of marriages, 
Table II. Single factor analysis of the risk factors of HPV infection.

Variables HPV-po
Number of marriages
0

$\begin{array}{lr}0 & 70 \\ 1 & \end{array}$

$\geq 2$

Education level

Junior high school or below

High school or above

Married

Yes

No

82

History of cervical disease

Yes

384

No

250

Tuberculosis

Yes

No

630

History of STDs

Yes

25

No

25

History of smoking

No

609

303

Yes

331

Age of first menstruation

$\leq 11$ years

12-15 years

$\geq 16$ years

Menopause

Yes

No

541

Abortion

Yes

74

No

560

Number of abortions

$$
1
$$

2

184

237

$\geq 3$

212

Condom use

Yes

216

No

418

Number of sexual partners

1

2

500

91

$\geq 3$

42

Number of sexual partners in the past 5 years

$$
1
$$

2

$\geq 3$

Sexual intercourse outside marriage

Yes

No

583

10

93

133

1405

97

863

773

1445

191

982

653

8

1627

55

1580

904

731

297

1078

261

233

1402

231

1404

598

545

493

666

969

1430

140

66

1366

157

113

140

1496
OR value

$95 \%$ CI

ve cases

rente

(a)

3

$\begin{array}{cc}1 & \\ 0.632^{\mathrm{a}} & 0.408-1.001 \\ 1.786^{\mathrm{a}} & 1.022-3.369\end{array}$

3

$0.369^{\mathrm{a}} \quad 0.304-0.601$

(2)

$1 \quad 0.854 \quad 0.534-1.313$

2

$3 \quad 1.019 \quad 0.765-1.343$

8

1.019

$0.765-1.343$

55

$1.289 \quad 0.231-7.083$

$\begin{array}{cc}1 & \\ 1.187 & 0.587-2.402\end{array}$

4

$1.349^{\mathrm{a}} \quad 1.039-1.771$

7

$\begin{array}{lll}1.133 & 0.791-1.622 \\ 1.041 & 0.641-1.649\end{array}$

$\begin{array}{ccc} & & \\ & 1 & \\ 02 & 1.032 & 0.712-1.523\end{array}$

$\begin{array}{ccc}1 & \\ 231 & 0.811 & 0.532-1.201\end{array}$

98

5

93

$\begin{array}{ll}1.415^{\mathrm{a}} & 1.024-1.961 \\ 1.406^{\mathrm{a}} & 1.001-1.972\end{array}$

$1.001-1.972$

66

(3)


Table II. Continued.

\begin{tabular}{|c|c|c|c|c|}
\hline Variables & HPV-positive cases & HPV-negative cases & OR value & $95 \% \mathrm{CI}$ \\
\hline \multicolumn{5}{|l|}{ Age at first sexual intercourse } \\
\hline$\leq 18$ years & 129 & 295 & 1 & \\
\hline 19-22 years & 224 & 668 & 0.761 & $0.524-1.121$ \\
\hline$\geq 23$ years & 280 & 673 & 0.931 & $0.637-1.336$ \\
\hline \multicolumn{5}{|l|}{ Occupation } \\
\hline Community residents & 91 & 623 & 1 & \\
\hline White-collar workers & 64 & 461 & 1.902 & $0.831-1.743$ \\
\hline Migrant workers & 134 & 409 & $3.288^{\mathrm{a}}$ & $2.241-4.851$ \\
\hline Workers in service industry & 171 & 316 & $3.995^{\mathrm{a}}$ & $2.473-5.247$ \\
\hline
\end{tabular}

${ }^{\text {a }}<0$ 0.05. HPV, human papillomavirus; OR, odds ratio; CI, confidence interval; STDs, sexually transmitted diseases.

Table III. Multiple factor analysis of the risk factors of HPV infection.

$95 \%$ CI for $\operatorname{Exp}(\mathrm{B})$

\begin{tabular}{lcccccccc}
\cline { 7 - 9 } Factor & $\mathrm{B}$ & $\mathrm{SE}$ & Wald & $\mathrm{df}$ & P-value & $\operatorname{Exp}(\mathrm{B})$ & Lower & Upper \\
\hline Education level & -0.871 & 0.152 & 32.233 & 1 & 0.000 & 0.423 & 0.301 & 0.559 \\
Number of sexual partners & 0.326 & 0.133 & 5.923 & 1 & 0.012 & 1.379 & 1.062 & 1.791 \\
Condom use & -0.372 & 0.146 & 6.238 & 1 & 0.009 & 0.681 & 0.509 & 0.919 \\
Occupation & 0.453 & 0.061 & 51.949 & 1 & 0.000 & 1.581 & 1.389 & 1.778
\end{tabular}

B, partial regression coefficient; Wald, Wald $\chi^{2}$ test; df, degree of freedom; Exp (B), exponentiation of the B coefficient; CI, confidence interval; HPV, human papillomavirus.

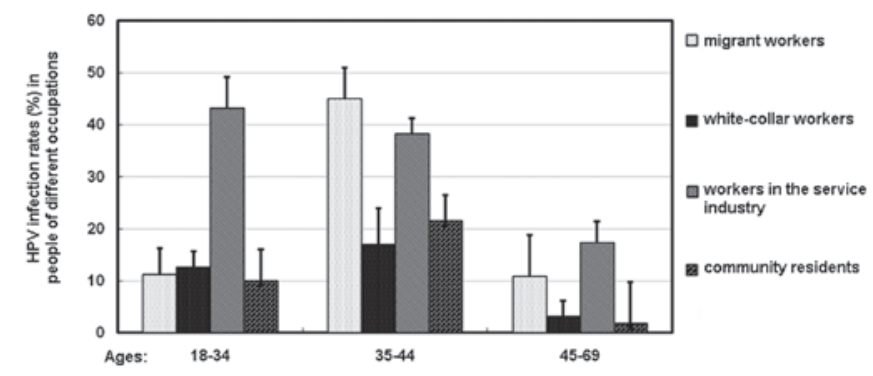

Figure 2. Human papillomavirus (HPV) infection rates of patients (18-69 years old) with different occupations in Urumqi Saybagh district, China. All subjects underwent gynecological examination and cervical secretion was collected A genome extraction kit was used for DNA extraction. Polymerase chain reaction (PCR) amplification using the ABI7300 PCR amplification instrument and hybridization were performed. For all the subjects with abnormal liquid-based cytology results, colposcopy and biopsy were performed. Data are presented as mean \pm standard error of the mean (SEM).

education level, smoking history, number of abortions, use of condoms, number of sexual partners, number of sexual partners in the past five years and occupation were associated with HPV infection rates $(\mathrm{P}<0.05)$.

To further confirm the results, logistic regression model analysis was performed on variables with statistical significance in univariate analysis ( $\alpha$ in $=0.05, \alpha$ out $=0.10$; in, the inclusion criteria; out, the exclusion criteria). The data in Table III shows that education level, number of sexual partners, condom use and occupation were incorporated into the model. The results suggest that education level and condom use were two protective factors of HPV infection, while the number of sexual partners and occupation were risk factors for HPV infection.

\section{Discussion}

In this study, a stratified cross-sectional survey was performed to conduct genotyping detection of 21 types of HPV in a large sample. The distribution of HPV-DNA subtypes varies in different districts and ethnic groups. de Sanjosé et al (11) conducted a meta-analysis of HPV infection in six regions of the world in 157,879 females without cervical lesions (by cytological diagnosis). The HPV infection rate was $\sim 10.4 \%$.

The prevalence of HPV infection varies significantly. The HPV infection rates are as follows: $22.1 \%$ in Africa, $20.4 \%$ in the central United States and Mexico, $11.3 \%$ in North America, $8.1 \%$ in Europe and $8.0 \%$ in Asia (11). Bell et al (12) determined the mean HPV infection rate of 287 American Indian females (by PCR assay) to be $21.25 \%$. Of these, $67.2 \%$ was high-risk HPV infection and $41 \%$ was multiple infection, and the common HPV infection subtypes were HPV-59, 39 and 73. In China, Li and Dai (13) conducted a study to determine the HPV infection rate in three regions of Shanxi, Shenyang and Shenzhen. The authors identified that the HPV infection 
rate was $16.1 \%$ and $\mathrm{HPV}-16$ is the most common virus type, followed by HPV-58, 52 and 18 (13). In the present study, we identified that the HPV infection rate in Urumqi, Xinjiang is $20.27 \%$, higher than that of Europe and the results of the study by Li and Dai, and similar to that of American Indian females, which may be explained by the fact that Xinjiang is an area with a high incidence of cervical cancer. We demonstrated that the common subtypes in Urumqi, Xinjiang are HPV-16, 58, 52 and 18, which is similar to the results of the study by $\mathrm{Li}$ and Dai (8). The existing vaccines are only effective for subtypes 16 and 18; however, 58 and 52 are also highly prevalent. Therefore, a customized HPV vaccine is required for the prevention and treatment of cervical lesions in Chinese females.

In a number of developed countries, including Spain and South Korea, the HPV infection peak usually occurs in young individuals under the age of 25 years and it declines sharply as age increases. In certain areas of South America, the HPV infection rate of 35-54-year-olds is lower than that of younger individuals ( $<25$ years) and elderly individuals ( $>55$ years). In countries with a high incidence of cervical cancer, including India and Nigeria, the HPV infection rate of individuals aged $35-54$ years is higher than that in younger individuals ( $<25$ years) and elderly individuals (>55 years) (14). In the present study, we identified that that the infection rate of individuals aged 35-44 years is significantly higher than that in younger individuals (18-34 years) and elderly individuals ( $>45$ years). The infection rate of HPV in the younger individuals was significantly higher than that in the elderly group. Our results are similar to data in India and Nigeria, but different from data in developed countries including Spain and South Korea. The high infection rate in the 35 to 44 age group was possibly due to the reason that females in that age group are sexually active.

HPV infection rates were increased with aggravated cervical lesions. The HPV infection rate in cervical cancer was $100 \%$, demonstrating again the correlation between cervical cancer and HPV infection. HPV infection rates in different age groups with different professions were also examined. The HPV infection rate of workers in service industries was significantly higher than the infection rates of white-collar workers, community residents and migrant workers. Among workers in service industries, the HPV infection rate of females aged 18-34 years was higher than that of those aged 35-44 and 45-69 years. This indicates that female workers in service industries aged 18-34 years are the most sexually active. A lack of health protection is a possible reason for the high HPV infection rate.

In this study, we identified that the number of marriages, education level, smoking history, number of abortions, condom use, number of sexual partners, number of sexual partners in the past five years and occupation are risk factors for HPV infection. A large number of epidemiological studies have shown that a premature sex life, multiple sexual partners, unprotected sexual intercourse and abortion increase the risk of cervical cancer (15-17). One study reported that as the smoking time increases, the risk of cervical cancer increases (18). In the current study, smoking was also observed to be a risk factor for HPV infection. The use of condoms avoids cross-infection, thus reducing the prevalence of HPV.

In conclusion, the infection rate of HPV is high in Urumqi, Xinjiang, with HPV-16, 58, 52 and 18 as the common genotypes. Since females aged 35-44 years have the highest rate of infection, screening of cervical lesions and health habit guidance should be performed.

\section{Acknowledgements}

This study was supported by the National Natural Science Foundation of China (grant no. 81101555) and the Project of the Opening Laboratory of Infection and Immunity (grant no. GRMY-2011-05), operated by Institute Pasteur of Shanghai, Chinese Academy of Sciences and the First Affiliated Hospital, Xinjiang Medical University.

\section{References}

1. Louie KS, de Sanjose S, Diaz M, et al: Early age at first sexual intercourse and early pregnancy are risk factors for cervical cancer in developing countries. Br J Cancer 100: 1191-1197, 2009.

2. Lang J: Problems in connection with diagnosis and treatment of cervical lesions. Zhonghua Fu Chan Ke Za Zhi 36: 261-263, 2001 (In Chinese).

3. Weinstein LC, Buchanan EM, Hillson C, et al: Screening and prevention: cervical cancer. Prim Care 36: 559-574, 2009.

4. Cuzick J, Arbyn M, Sankaranarayanan R, et al: Overview of human papillomavirus-based and other novel options for cervical cancer screening in developed and developing countries. Vaccine 26 (Suppl 10): K29-K41, 2008.

5. Cruickshank ME: The role of human papillomavirus in risk management. Rev Gynaecol Practice 3: 292-233, 2003.

6. Walboomers JM, Jacobs MV, Manos MM, et al: Human papillomavirus is a necessary cause of invasive cervical cancer worldwide. J Pathol 189: 12-19, 1999.

7. Pilch H, Günzel S, Schäffer U, et al: The presence of HPV DNA in cervical cancer: correlation with clinico-pathologic parameters and prognostic significance: 10 years experience at the Department of Obstetrics and Gynecology of the Mainz University. Int J Gynecol Cancer 11: 39-48, 2001.

8. Stevens MP, Garland SM, Tan JH, et al: HPV genotype prevalence in women with abnormal pap smears in Melbourne, Australia. J Med Virol 81: 1283-1291, 2009.

9. Wu K, Fu Y and Jiang H: Molecular epidemiology analysis of cervical HPV in women of childbearing age in Guangzhou, China. Guangdong Med 25: 386-391, 2004 (In Chinese).

10. Prandi S, Beccati D, De Aloysio G, et al: Applicability of the Bethesda System 2001 to a public health setting. Cancer 108: 271-276, 2006.

11. de Sanjosé S, Diaz M, Castellsagué X, et al: Worldwide prevalence and genotype distribution of cervical human papillomavirus DNA in women with normal cytology: a meta-analysis. Lancet Infect Dis 7: 453-459, 2007.

12. Bell MC, Schmidt-Grimminger D, Patrick S, Ryschon T, Linz L and Chauhan SC: High prevalence of human papillomavirus infection in American Indian women of the Northern Plains. Gynecol Oncol 2: 236-241, 2007 (In Chinese).

13. Li N and Dai M: Human papillomavirus infection in China: a multi-centric cross-sectional study. Chinese Journal of Disease Control and Prevention 12: 411-415, 2008.

14. Franceschi S, Herrero R, Clifford GM, et al: Variations in the age-specific curves of human papillomavirus prevalence in women worldwide. Int J Cancer 119: 2677-2684, 2006.

15. Zeng ZP, Chen F, Liu B, et al: Studies of risk factors of cervical cancers in Yangcheng County, Shanxi Province, China. Tumor Prevention Research 31: 178-181, 2004.

16. Zhao FH, Ma JF, Qiao YL, et al: Association between high-risk human papillomavirus DNA load and cervical intraepithelial lesion. Zhonghua Liu Xing Bing Xue Za Zhi 25: 921-924, 2004 (In Chinese).

17. Zhao FH, Forman MR, Belinson J, et al: Risk factors for HPV infection and cervical cancer among unscreened women in a high-risk rural area of China. Int J Cancer 118: 442-448, 2006.

18. Deacon JM, Evans CD, Yule R, et al: Sexual behaviour and smoking as determinants of cervical HPV infection and of CIN3 among those infected: a case-control study nested within the Manchester cohort. Br J Cancer 83: 1565-1572, 2000. 